\title{
BMJ
}

\section{Non-specific effects of standard measles vaccine at 4.5 and 9 months of age on childhood mortality: randomised controlled trial}

\author{
Peter Aaby, professor, ${ }^{1,2}$ Cesário L Martins, clinician, ${ }^{1}$ May-Lill Garly, senior researcher, ${ }^{1,2}$ Carlito Balé, clinician, ${ }^{1}$ \\ Andreas Andersen, statistician, ${ }^{1,2}$ Amabelia Rodrigues, research director, ${ }^{1}$ Henrik Ravn, senior statistician, ${ }^{1,2}$ \\ Ida M Lisse, senior registar, ${ }^{3}$ Christine S Benn, senior researcher, ${ }^{1,2}$ Hilton C Whittle, professor ${ }^{4}$
}

\section{${ }^{1}$ Bandim Health Project, Indepth Network, Bissau, Guinea-Bissau \\ ${ }^{2}$ Bandim Health Project, Danish Epidemiology Science Centre, Statens Serum Institut, 2300 \\ Copenhagen S, Denmark \\ ${ }^{3}$ Department of Pathology, Herlev University Hospital, 2730 Herlev, Denmark \\ ${ }^{4} \mathrm{MRC}$ Laboratories, Fajara, POB 273, Gambia \\ Correspondence to: P Aaby p.aaby@bandim.org}

Cite this as: BMJ 2010;341:C6495 doi:10.1136/bmj.c6495

\section{ABSTRACT}

Objective To examine in a randomised trial whether a $25 \%$ difference in mortality exists between 4.5 months and 3 years of age for children given two standard doses of Edmonston-Zagreb measles vaccines at 4.5 and 9 months of age compared with those given one dose of measles vaccine at 9 months of age (current policy).

Design Randomised controlled trial.

Setting The Bandim Health Project, Guinea-Bissau, which maintains a health and demographic surveillance system in an urban area.

Participants 6648 children aged 4.5 months of age who had received three doses of diphtheria-tetanus-pertussis vaccine at least four weeks before enrolment. A large proportion of the children ( $80 \%$ ) had previously taken part in randomised trials of neonatal vitamin $\mathrm{A}$ supplementation.

Intervention Children were randomised to receive Edmonston-Zagreb measles vaccine at 4.5 and 9 months of age (group A), no vaccine at 4.5 months and Edmonston-Zagreb measles vaccine at 9 months of age (group B), or no vaccine at 4.5 months and Schwarz measles vaccine at 9 months of age (group C).

Mainoutcomemeasure Mortality rate ratio between 4.5 and 36 months of age for group A compared with groups $B$ and $C$. Secondary outcomes tested the hypothesis that the beneficial effect was stronger in the 4.5 to 9 months age group, in girls, and in the dry season, but the study was not powered to test whether effects differed significantly between subgroups.

Results In the intention to treat analysis of mortality between 4.5 and 36 months of age the mortality rate ratio of children who received two doses of Edmonston-Zagreb vaccine at 4.5 and 9 months of age compared with those who received a single dose of Edmonston-Zagreb vaccine or Schwarz vaccine at 9 months of age was 0.78 (95\% confidence interval 0.59 to 1.05 ). In the analyses of secondary outcomes, the intention to treat mortality rate ratio was 0.67 ( 0.38 to 1.19 ) between 4.5 and 9 months and 0.83 ( 0.83 to 1.16 ) between 9 and 36 months of age. The effect on mortality between 4.5 and 36 months of age was significant for girls (intention to treat mortality rate ratio $0.64(0.42$ to 0.98$))$, although this was not significantly different from the effect in boys $(0.95$ ( 0.64 to 1.42)) (interaction test, $P=0.18$ ). The effect did not differ between the dry season and the rainy season. As neonatal vitamin A supplementation is not WHO policy, the analyses were done separately for the 3402 children who did not receive neonatal vitamin $\mathrm{A}$. In these children, the two dose Edmonston-Zagreb measles vaccine schedule was associated with a significantly lower mortality between 4.5 and 36 months of age (intention to treat mortality rate ratio 0.59 (0.39 to 0.89$)$ ). The effect was again significant for girls but not statistically significant from the effect in boys. When measles cases were censored, the intention to treat mortality rate ratio was 0.65 (0.43 to 0.99).

Conclusions Although the overall effect did not reach statistical significance, the results may indicate that a two dose schedule with Edmonston-Zagreb measles vaccine given at 4.5 and 9 months of age has beneficial nonspecific effects on children's survival, particularly for girls and for children who have not received neonatal vitamin A. This should be tested in future studies in different locations.

Trial registration Clinical trials NCT00168558.

\section{INTRODUCTION}

When measles vaccine was introduced at demographic surveillance sites in Africa in the late 1970s and early 1980 s, several studies reported major reductions in childhood mortality of $40 \%$ or more. ${ }^{1-7}$ Population based studies in Bangladesh and Haiti gave similar results. ${ }^{8-10}$ The effect was much larger than expected, as measles infection caused much less than $40 \%$ of the deaths in these communities. These observations suggested that measles vaccine may have non-specific beneficial effects that provide protection against infections other than measles. ${ }^{11}$

An implication of these observations was that lowering the age of measles vaccination might be beneficial. The World Health Organization set the age of measles vaccination at 9 months to minimise interference from maternal antibodies. ${ }^{12}$ However, 
medium titre and high titre Edmonston-Zagreb measles vaccine can be used effectively even in the presence of maternal antibodies. ${ }^{1314}$ In the mid-1980s, we therefore did several trials in the Gambia, GuineaBissau, and Senegal, in which children were vaccinated at 4-5 months of age with medium titre or high titre Edmonston-Zagreb measles vaccine. ${ }^{13-18}$ WHO recommended the high titre version of the Edmonston-Zagreb vaccine for routine use in 1989. However, the West African trials surprisingly showed that early Edmonston-Zagreb vaccination at 4-5 months of age was associated with twofold higher mortality in girls. ${ }^{1819}$ After these results were confirmed in Haiti, ${ }^{20}$ WHO rescinded its recommendation for the use of high titre measles vaccine in $1992 .{ }^{21}$ High titre measles vaccine was fully protective against measles, and these trials therefore showed that high titre measles vaccine had non-specific and sex differential effects on children's survival. ${ }^{19}$ Nevertheless, few attempts were made to explain these effects, and the major funding institutions invested in the development of new measles vaccines.

We pursued the hypothesis that standard titre measles vaccine given in a two dose schedule at 6 and 9 months of age could overcome the interference from maternal antibodies and be fully protective against measles infection and could have beneficial nonspecific effects on overall survival between 6 and 9 months of age. ${ }^{21-25}$ The results of our first randomised trial of two dose vaccine were disappointing; compared with inactivated polio vaccine, which was used as a control vaccine, the mortality rate ratio for early measles vaccine at 6 months of age was 0.90 (95\% confidence interval 0.67 to 1.22 ) between 6 and 9 months of age. ${ }^{25}$ However, the study design had not taken account of the interaction with diphtheria-tetanus-pertussis (DTP) vaccine. DTP vaccine has been associated with increased mortality in girls. ${ }^{25}$ Among girls who had received all three doses of DTP vaccine before enrolment and were therefore unlikely to receive additional doses of DTP vaccine during follow-up, measles vaccine tended to have a beneficial effect compared with inactivated polio vaccine. ${ }^{25}$ However, girls vaccinated with measles vaccine who were missing doses of DTP vaccine at enrolment and were likely to receive additional doses during follow-up had twofold higher mortality than did girls who had received three doses of DTP vaccine before enrolment. ${ }^{25}$ We speculated that DTP vaccine after measles vaccine could also have been the explanation for the increased mortality in girls after high titre measles vaccine ${ }^{19}$ : high titre measles vaccine had been administered early, and most children had received $\mathrm{DTP}$ /inactivated polio vaccine after high titre measles vaccine. When we reexamined the original dataset, we saw no excess mortality in girls among children who had not received DTP/inactivated polio vaccine after high titre measles vaccine but a twofold higher mortality in girls among children who had received DTP/inactivated polio vaccine after high titre measles vaccine. ${ }^{19}$
On the basis of these observations, we started a second trial of two dose vaccine in 2003, randomising children to a standard dose of Edmonston-Zagreb measles vaccine at 4.5 and 9 months of age or no vaccine at 4 . 5 months and measles vaccine at 9 months of age. ${ }^{2627}$ To prevent the interference from DTP vaccinations administered after measles vaccine, only children who had received all three doses of DTP vaccine were enrolled in the trial. To ensure that an interval of at least four weeks existed between the third DTP vaccine and measles vaccine, the earliest we could vaccinate against measles was at 4.5 months of age. Our hypothesis was that early measles vaccine would have a beneficial effect, particularly for girls. We aimed to measure the effect of measles vaccine at 4.5 and 9 months of age on survival between 4.5 months and 3 years of age, compared with no vaccine at 4.5 months of age and either Edmonston-Zagreb or Schwarz measles vaccine at 9 months of age as currently recommended by WHO. All participants have now reached 3 years of age, and we therefore report the effect of early measles vaccination on overall mortality between 4.5 months and 3 years of age.

\section{METHODS}

\section{Setting and study population}

The trial took place in Guinea-Bissau in the Bandim Health Project's study area, which covers six districts with approximately 102000 inhabitants - that is, 30\% of the population of the capital Bissau. ${ }^{28}$ All residents have an identification number in the census files, and information on the place of residence can be retrieved from these files, along with socioeconomic and demographic information. All houses are visited every month to register new pregnancies and births. All children are visited at home every three months until the age of 3 years; at these visits, we collect information on breastfeeding status, infections, hospital admissions, vaccination status, living with the mother, and ownership of pigs. Three health centres in the project area provide routine vaccinations. At the beginning of this trial, the coverage for the third DTP vaccine was $89 \%$ and that for measles vaccine was $88 \%$ among children aged $12-23$ months of age. ${ }^{29}$

During the trial period from 2003 to 2009, many government driven health intervention campaigns occurred, as is now usual in Guinea-Bissau and other West African countries. We had field assistants who accompanied all the campaign teams in the study area to monitor who received the interventions (table 1). Two measles vaccination campaigns took place in May 2006 and July 2009. With permission from the Ministry of Health, the study participants were excluded from these campaigns (see web table A). Participants in the trial could have taken part in other randomised trials in the area (table 1 and web table A). ${ }^{30-32}$ Thus, even though the two dose measles trial was randomised, we needed to examine in subanalyses the effects that these other interventions may have had on the main intervention. 
Table 1| Background factors for early two dose measles vaccine (group A) and measles vaccine at 9 months (groups $B+C$ ). Values are percentages (numbers) unless stated otherwise

Early two dose measles vaccine (group A; $n=2129$ )

Measles vaccine at 9 months (groups $B$ and $C ; n=4288$ )

Demographic factors

Median (interquartile range) age at enrolment (months)

Median (interquartile range) age of mother (years)

Bandim district

Male sex

Twins

Pepel ethnicity

Mother died (No)

Risk factors at enrolment

Not breast fed at 4.5 months

Pigs in household

No of people/bed

No of people/sleeping room

Toilet inside house

Functioning electricity

Hospital admission before enrolment

\begin{tabular}{cc}
\hline $4.8(4.7-5.2)$ & $4.8(4.7-5.2)$ \\
\hline $25(21-29)$ & $25(20-29)$ \\
\hline $42(898)$ & $42(1794)$ \\
\hline $51(1084)$ & $50(2151)$ \\
\hline $3.6(77)$ & $3.4(145)$ \\
$31(662)$ & $29(1224)$ \\
\hline
\end{tabular}

3

8

Morbidity and anthropometry at enrolment

\begin{tabular}{|c|c|c|}
\hline Fever & $8.4(178)$ & $9.4(403)$ \\
\hline Diarrhoea & $4.7(101)$ & $4.4(188)$ \\
\hline Mean respiratory frequency & 42 & 43 \\
\hline BCG scar & $86(1832)$ & $86(3673)$ \\
\hline Have chloroquine at home & $35(745)$ & $33(1408)$ \\
\hline Mean (SD) weight (g) & $7153(988)$ & $7134(998)$ \\
\hline Mean (SD) arm circumference (mm) & $142(12)$ & $142(12)$ \\
\hline Mean (SD) height (cm) & $64.1(2.8)$ & $64.1(2.8)$ \\
\hline Mean (SD) mother's arm circumference (mm) & $275(34)$ & $274(34)$ \\
\hline \multicolumn{3}{|l|}{ Participation in other trials and campaigns* } \\
\hline LBW trial & $6.3(134)$ & $6.3(268)$ \\
\hline First vitamin A trial & 34 (719) & $34(1466)$ \\
\hline Second vitamin A trial & $44(944)$ & $44(1883)$ \\
\hline DTP booster trial & $52(1109)$ & $53(2269)$ \\
\hline Measles vaccine campaign 2006 & $3.1(65)$ & $3.2(139)$ \\
\hline Measles vaccine campaign 2009 & $1.6(35)$ & $1.4(60)$ \\
\hline Bed net distribution 2006 & $44(928)$ & $42(1817)$ \\
\hline Bed net distribution 2007 & $41(870)$ & $38(1637)$ \\
\hline Bed net impregnation 2006 & $24(516)$ & $23(1004)$ \\
\hline Bed net impregnation 2007 & $26(564)$ & $26(1103)$ \\
\hline OPV campaign 2004 & $40(844)$ & 39 (1663) \\
\hline OPV campaign 2005 & $59(1251)$ & $58(2507)$ \\
\hline VAS campaign 2004 & $26(553)$ & $25(1077)$ \\
\hline VAS campaign 2005 & $46(971)$ & 45 (1949) \\
\hline First VAS campaign $2006 \dagger$ & $56(1187)$ & $56(2404)$ \\
\hline Second VAS campaign 2006 & $38(818)$ & 37 (1597) \\
\hline First VAS campaign 2007 & $48(1016)$ & 47 (1994) \\
\hline Second VAS campaign 2007 & 34 (729) & $35(1503)$ \\
\hline First VAS campaign 2008 & $22(458)$ & 23 (989) \\
\hline Second VAS campaign 2008 & $43(918)$ & $43(1854)$ \\
\hline First VAS campaign 2009 & $27(574)$ & 27 (1155) \\
\hline
\end{tabular}

DTP= diphtheria-tetanus-pertussis; $L B W=$ low birth weight; $O P V=$ oral polio vaccine; $V A S=v i t a m i n ~ A$ supplementation.

*All campaigns in study population were monitored to assess possible interactions; field assistants accompanied all campaign teams in study area.

†From 2006 onwards vitamin A supplementation campaigns have included treatment with mebendazole for children aged 1-4 years.

\section{Objectives and study design}

The primary objective of this study was to measure the effect of different strains and schedules of measles vaccination on overall mortality between 4.5 and 36 months of age. The sample size was based on the hypothesis that we should be able to measure a $25 \%$ difference in mortality between early measles vaccination and the current policy of measles vaccination at 9 months of age. The secondary objectives were to measure mortality between 4.5 and 9 months of age and between 9 and 36 months of age and to determine the possible effect of sex and season. We hypothesised that the non-specific beneficial effects would be strongest for girls and in the dry season. However, we could not power the study to test a significant difference between girls and boys and between dry and rainy seasons.

The trial included three arms: two dose measles vaccination providing standard dose Edmonston-Zagreb measles vaccine at 4.5 and 9 months of age (group A), no vaccine at 4.5 months and standard dose Edmonston-Zagreb measles vaccine at 9 months of age (group B), and no vaccine at 4.5 months and standard dose Schwarz measles vaccine at 9 months of age (group C). All children were enrolled and randomised at 4 . 5 months of age. Because a condition for entering the trial was that the children had received the third DTP vaccine at least four weeks before enrolment, the children in groups B and C had the third DTP vaccine as their most recent vaccination between 4.5 and 9 months of age. We have previously reported the clinical protection of one dose of measles vaccine at 4 . 5 months of age $\mathrm{e}^{26}$; the first dose of measles vaccine at 4 . 5 months of age was highly protective against clinical measles infection and $100 \%$ protective against hospital admission for measles (95\% confidence interval $46 \%$ to $100 \%)$ and death from measles $(-42 \%$ to $100 \%)$. By 24 months of age, all the 925 children tested in the study had responded with specific antibodies to measles vaccination; $97 \%$ in group A, $99 \%$ in group $\mathrm{B}$, and $99 \%$ in group $\mathrm{C}$ had protective levels of measles antibodies (unpublished data).

The WHO immunisation programme uses standard dose Edmonston-Zagreb and Schwarz measles vaccines interchangeably. However, if vaccines have non-specific effects, ${ }^{11} 19$ these could differ between two strains of measles vaccine with very different biological qualities, including that Edmonston-Zagreb is more immunogenic than Schwarz in the presence of maternal antibodies. ${ }^{23}$ We therefore intended to compare the effect on children's survival of these two strains by comparing groups B and C. We used the early two dose measles vaccination schedule to examine whether effective vaccination at 4.5 months and 9 months of age with long term maintenance of measles antibodies was possible and to study whether measles vaccine compared with the third DTP vaccine had non-specific beneficial effects for child health by comparing group A with groups B and C. We hypothesised that a beneficial effect would be particularly important for girls. We present the results of this analysis here. 
We called the children in groups $\mathrm{B}$ and $\mathrm{C}$ for an additional visit at 18 months and randomised them to an additional dose of the same strain of measles vaccine or no additional dose to examine whether the additional dose had an effect on the long term maintenance of measles antibody concentrations. The additional dose had no effect on antibody concentrations (unpublished data). We found no difference in mortality between 18 months and 36 months of age in children who received an additional dose and those who did not (fig 1). We have therefore not further divided the children in group $\mathrm{B}$ and $\mathrm{C}$ into those who received or did not receive an additional dose of measles vaccine.

\section{Sample size}

We based the sample size on the calculation that, with a power of $80 \%$, a significance level of $5 \%$, and an annual mortality rate of 50 per 1000 person years, we needed 4360 person years in each group to detect a $25 \%$ difference in mortality from 4.5 to 36 months of age between any two of the three groups. With an expected $10 \%$ loss to follow-up and an average follow-up of 30 months, we needed 1918 children in each arm of the study.
Owing to additional immunological studies, we enlarged the cohort to include 6648 children. As all children were treated according to the original protocol until 3 years of age, we analysed mortality for the complete cohort.

\section{Enrolment, informed consent, and randomisation}

Study procedures have been described in detail in a previous paper. ${ }^{26} \mathrm{We}$ identified newborn infants in the Bandim Health Project registration system and reminded mothers to bring their children for DTP vaccination at 6,10 , and 14 weeks of age. Enrolment in the measles vaccination trial occurred at 4.5 months of age. To facilitate access of the local populations, enrolment was organised by the same team of physician, nurses, and field workers on different weekdays at the three local health centres. The mothers or guardians of children presenting at the local health centre received an oral and a written explanation of the study from a doctor. They were told that which measles vaccine strain or vaccination strategy was best was not known. At each visit to the health centre, the doctor did a medical examination.

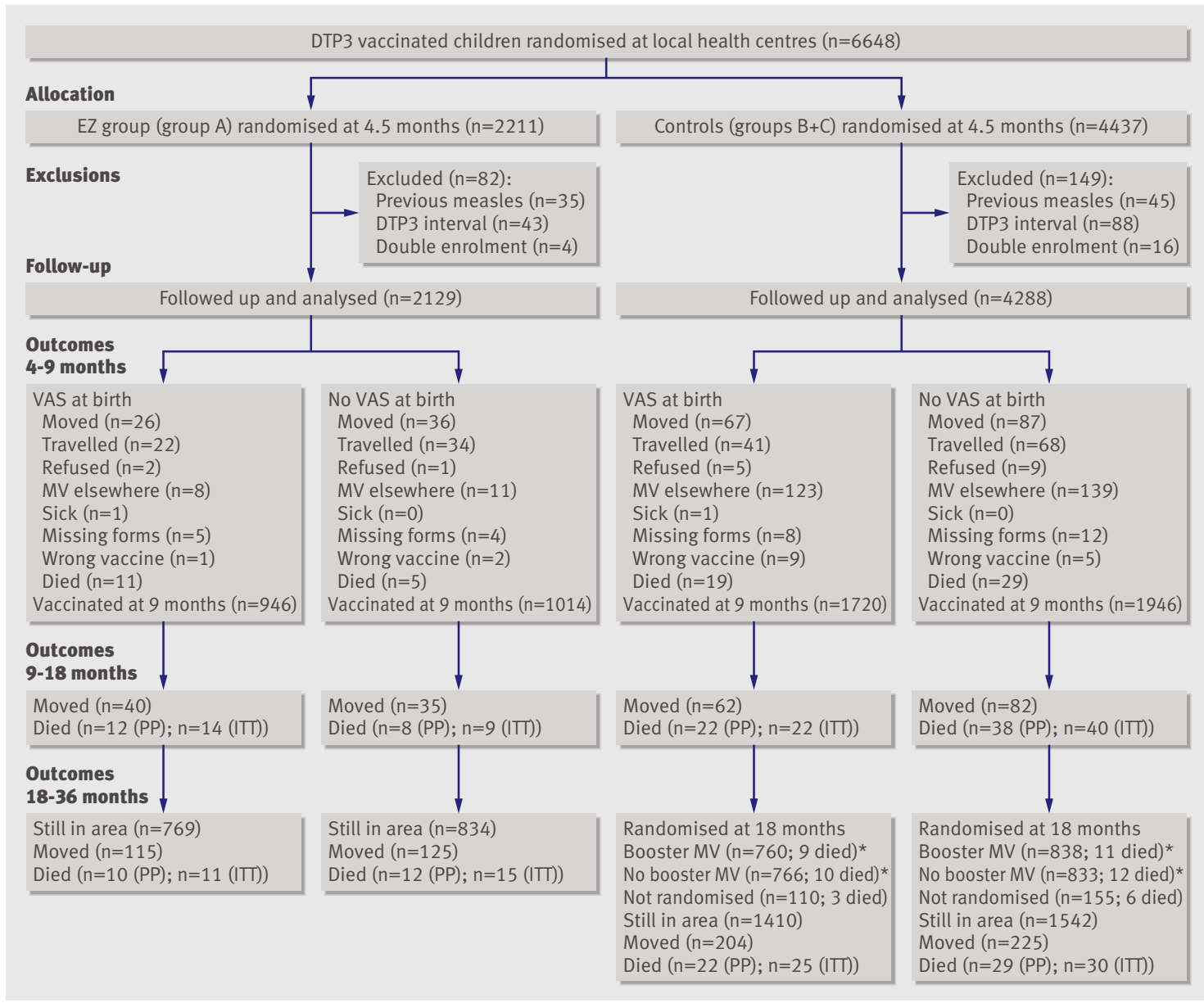

Fig 1| Trial diagram for early two dose measles vaccination trial. DTP3=third diphtheria-tetanus-pertussis vaccine; $E Z=E d m o n s t o n-Z a g r e b$ vaccine; ITT=intention to treat analysis; $M V=$ measles vaccine; $P P=$ =per protocol analysis; VAS=vitamin A supplementation. ${ }^{*}$ Children in groups $B$ and $C$ were randomised to receive or not receive a booster dose of same measles vaccine at 18 months of age; deaths in parenthesis are number of deaths between randomisation at 18 months of age and end of the study; these deaths are included in total for group 
If they agreed to participate, the mothers or guardians were asked to select an envelope with the allocation number that defined to which group the child belonged. We randomised children to one of three equal sized groups by using block randomisation with 24 envelopes per bag. Block randomisation was organised within the whole study area and not by health centre. Study participants had access to free consultations at the local health centres and to essential drugs free of charge.

\section{Intervention}

Children randomised to group A received a standard titre Edmonston-Zagreb vaccine from the Serum Institute of India, Pune, India, at 4.5 and 9 months of age. At 9 months of age, the children in group $B$ received the same Edmonston-Zagreb measles vaccine, and children in group $\mathrm{C}$ received a Schwarz measles vaccine (Rouvax) from Pasteur Merieux, France.

\section{Masking}

As described previously, no placebo was given. ${ }^{26} \mathrm{We}$ have previously used inactivated polio vaccine as a control vaccine in trials of early measles vaccination. ${ }^{25}$ However, inactivated polio vaccine and other inactivated vaccines may increase the female to male mortality ratio, ${ }^{25}$ so we could not use a control vaccine if we were to measure the non-specific effects of measles vaccine. If we had used a placebo, some mothers in the control group might erroneously have believed that the child had received measles vaccine. As long as they remained in the study area this would matter little, as they would all be called for measles vaccination at 9 months of age. However, if they moved from the area they might not have gone to another health centre to seek measles vaccination. We therefore preferred to use no placebo. Mothers in the control group were told that their children would not receive measles vaccine at enrolment, but that they should receive measles vaccine at 9 months of age.

\section{Measurement of mortality}

Mortality between 4.5 and 9 months of age-All children were invited back to the health centre at 9 months of age. If the children were not at home or were travelling, we kept calling the children until they were 18 months of age.

Mortality between 9 and 36 months of age-All children were invited back to the health centre at 24 months of age, and all children were visited at home after 36 months of age by the Bandim Health Project demographic surveillance system.

At these visits we obtained information about whether the children had died or moved: we followed children who moved within the study area to their new address, and we censored children who moved out of the area in the survival analysis at the time of moving. Mothers and their children travel frequently to visit family in the rural areas or to take part in the harvest of cashew nuts. However, information on survival is always available; if a child dies in the rural areas, the family in Bissau is informed immediately.

\section{Change in context: measles epidemic}

A measles outbreak began during the early phase of the trial in October 2003 and lasted until May 2004. This epidemic was unexpected because Bandim Health Project has kept a very high coverage for measles vaccination for children under 3 years of age for many years in this community. ${ }^{26}$ However, the coverage of vaccination had generally declined in the city, and many older and presumably unvaccinated children had moved in from rural areas. The epidemic and measles surveillance during the epidemic has been described elsewhere,${ }^{2629}$ and we tested the efficacy of measles vaccine at 4.5 months of age during the epidemic. ${ }^{26}$ During the study, 110 children were diagnosed as having measles: 71 cases were confirmed by serology, 10 did not have complete serology but were diagnosed as definite measles by clinicians, and 29 were diagnosed by the mother. For this study, we used the data on measles cases to censor for measles infection in the survival analysis to obtain an estimate of the effect of measles vaccine on non-measles related mortality. By censoring all cases of measles at the time of measles infection, we excluded from the comparison not only deaths from acute measles but also potential long term excess mortality after measles infection.

\section{Statistical analyses}

We entered data daily. We proofread important variables and assured consistency with the general registration system. We used Stata version 10 for the statistical analyses. For mortality, we present deaths and observation time together with hazard rate ratios and Wald 95\% confidence intervals estimated from a Cox proportional hazards model with age as the underlying time variable. $\mathrm{P}$ values were calculated from Wald tests. As in previous survival analyses, we label these mortality hazards rate ratios as mortality rate ratios. Using time since inclusion as the underlying time made virtually no difference to the results. We assessed the proportional hazards assumption graphically and tested it by using Schoenfeld residuals $(\mathrm{P}=0.70)$. We adjusted for age as inherent in the Cox model and for district by stratification. We used the Kaplan-Meier method to calculate accumulated mortality curves for groups $\mathrm{A}$ and $\mathrm{B}+\mathrm{C}$, both overall and stratified by birth cohort.

In the intention to treat analysis, children were followed from enrolment to 36 months of age or until censoring due to movement or death, irrespective of whether the child received measles vaccine at 9 months of age. In the per protocol analyses, we examined the mortality of all children from enrolment at 4 . 5 months of age to the 9 month vaccination and mortality between 9 and 36 months of age for the children who received measles vaccine at 9 months of age. As we compared different strains of measles vaccine, we needed to provide the measles vaccine to know what strain had been provided, so we censored children who had received measles vaccine elsewhere. An overall per protocol estimate between 4.5 and 36 months of age came from censoring children not receiving 
measles vaccine from us at 9 months of age. Hence, the intention to treat and per protocol analyses were the same between 4.5 and 9 months of age; the only difference was that children who did not receive measles vaccine from us at 9 months of age were included in the intention to treat but not in the per protocol analysis from 9 to 36 months of age.

\section{Exclusions}

In the initial phase of the trial, 80 children had had measles infection before enrolment at 4.5 months of age (five died). Furthermore, the protocol specified that children should be enrolled four weeks after the third DTP vaccination. However, in the first month, a programming error meant that 131 children were enrolled within 25 days of the third DTP vaccination (seven died). Furthermore, we excluded 18 children because they were enrolled twice (none died); this may happen if the child has been registered with two identification numbers because the mother and the father live in different places or because the child often stays with a grandparent; we excluded these children from the study. We excluded two children who had the wrong age recorded; they were both one year older than originally assumed (neither died). As in the previous analysis, ${ }^{26}$ we excluded these 231 children from the main analysis. A further 17 children received the wrong type of measles vaccine at 9 months of age and they have been censored in the analysis from the date of this vaccination (none died).

\section{RESULTS}

\section{Participants}

The number of children enrolled between August 2003 and April 2007 was 6648 (fig 1), and we included 6417 in the main analysis. Of these, 2129 were randomised to Edmonston-Zagreb measles vaccine at 4.5 and 9 months of age and 4288 were randomised to receive no vaccine at 4.5 months of age and EdmonstonZagreb or Schwarz measles vaccine at 9 months of age. The study ended in October 2009 when the last children became 3 years of age.

At 9 months of age, $64(1 \%)$ had died, 216 had moved, 167 were travelling throughout the period up to 18 months of age, 29 were not visited because of administrative errors in the printing of the 9 months examination forms, 281 had already received a measles vaccine elsewhere, and 17 refused to participate. A further 17 received the wrong vaccine and were therefore censored at 9 months of age (fig 1). Therefore, 5626 children took part in the last part of the study from 9 to 36 months of age. The proportion of children who had received measles vaccine elsewhere differed between group A and groups B and C (fig 1).

\section{Baseline data}

No major differences existed in the demographic, socioeconomic, and health related background factors between children included in the early two dose measles vaccine arm (group A) and the two groups with measles vaccine at 9 months of age (groups B + C) (table 1).

\section{Outcomes}

Main results

We found no difference in mortality between groups $\mathrm{B}$ and $\mathrm{C}$ from 4.5 to 36 months of age; the intention to treat and per protocol mortality rate ratios were 1.01 (95\% confidence interval 0.74 to 1.37$)$ and $0.99(0.72$ to 1.35) for Edmonston-Zagreb vaccine (78/2153 children) compared with Schwarz vaccine (81/2135 children). From 9 to 36 months of age after measles vaccination, the per protocol mortality rate ratio was 1.09 (0.75 to 1.59) for Edmonston-Zagreb vaccine (57/ 1841 children) compared with Schwarz vaccine (54/ 1825 children).

The effect of early two dose measles vaccine (group A) was similar when compared with EdmonstonZagreb measles vaccine at 9 months of age (group B: mortality rate ratios $0.79(0.57$ to 1.09$)$ for intention to treat analysis and $0.71(0.50$ to 1.00$)$ for per protocol analysis) or with Schwarz measles vaccine at 9 months of age (group C: mortality rate ratios 0.78 (0.57 to 1.08 ) for intention to treat and 0.69 (0.49 to 0.96$)$ for per protocol) (web tables B and C). As we found no differences in the pattern of mortality, we combined groups $\mathrm{B}$ and $\mathrm{C}$ as a control group for the comparison with early measles vaccine (group A) (table 2).

Table 2 shows that children assigned to an early two dose measles vaccine schedule between 4.5 and 36 months of age had insignificantly lower mortality in the intention to treat analysis (mortality rate ratio $0.78(0.59$ to 1.05$))$. In the per protocol analysis the mortality rate ratio was 0.70 (0.52 to 0.94$)$ (fig 2).

Secondary results

In both the intention to treat and per protocol analyses, the mortality rate ratio was 0.67 (0.38 to 1.19$)$ between 4.5 and 9 months. Between 9 and 36 months of age it was $0.83(0.59$ to 1.16$)$ in the intention to treat analysis and $0.71(0.50$ to 1.01$)$ in the per protocol analysis. In the intention to treat analysis, the effect was statistically significant for girls (mortality rate ratio 0.64 (0.42 to $0.98)$ ) but not for boys (mortality rate ratio 0.95 (0.64 to 1.42$)$ ), but the relative effect was not significantly different for girls compared with boys (female:male estimate ratio 0.68 ( 0.38 to 1.20$) ; \mathrm{P}=0.18)$. Similarly, in the per protocol analysis, the effect of early measles vaccination was significant for girls (mortality rate ratio 0.59 ( 0.38 to 0.91$)$ ) but not for boys (mortality rate ratio 0.82 (0.54 to 1.25)) (female:male estimate ratio 0.71 (0.39 to 1.30); $\mathrm{P}=0.27$ ) (table 2). After censoring for measles infection, the mortality rate ratio was 0.84 ( 0.63 to $1.12)$ in the intention to treat analysis and $0.74(0.55$ to 1.00$)$ in the per protocol analysis. The effect of early measles vaccination on mortality from 4.5 to 36 months of age was not significantly better for children enrolled during the dry season from December to May (mortality rate ratios 0.74 (0.48 to 1.13 ) for intention to treat and $0.62(0.40$ to 0.98$)$ for per protocol) than during the rainy season from June to November (mortality rate ratios 0.83 ( 0.56 to 1.22$)$ for intention to treat and 0.76 (0.51 to 1.14 ) for per protocol). 
Table $2 \mid$ Mortality rates and mortality rate ratios (with $95 \%$ confidence intervals) of recipients of early two dose measles vaccine (group A) compared with measles vaccine at 9 months (groups $B+C)$ among all children $(n=6417)$

\begin{tabular}{|c|c|c|c|c|c|c|}
\hline \multirow[b]{2}{*}{ Age interval } & \multicolumn{2}{|c|}{$\begin{array}{l}\text { ITT mortality per } 100 \text { person years } \\
\text { (deaths/person days) }\end{array}$} & \multirow[b]{2}{*}{$\begin{array}{l}\text { ITT mortality rate } \\
\text { ratio }(A /(B+C))\end{array}$} & \multirow[b]{2}{*}{$\begin{array}{l}\text { ITT mortality rate ratio } \\
(A /(B+C)) \text { (with censoring } \\
\text { for measles infection) }\end{array}$} & \multirow[b]{2}{*}{$\begin{array}{l}\text { PP mortality rate ratio } \\
\qquad(A /(B+C))\end{array}$} & \multirow[b]{2}{*}{$\begin{array}{l}\text { PP mortality rate ratio } \\
(A /(B+C)) \text { (with censoring } \\
\text { for measles infection) }\end{array}$} \\
\hline & $\begin{array}{l}\text { Early two dose measles } \\
\text { vaccine (group A) }\end{array}$ & $\begin{array}{l}\text { Measles vaccine } \\
\text { at } 9 \text { months } \\
\text { (groups B and C) }\end{array}$ & & & & \\
\hline \multicolumn{7}{|l|}{ 4.5-9 months: } \\
\hline Boys & $\begin{array}{c}2.6(10 / 142341) \\
(n=1084)\end{array}$ & $\begin{array}{c}2.7(21 / 282932) \\
(n=2151)\end{array}$ & $0.94(0.44$ to 2.01$)$ & $1.22(0.55$ to 2.70$)$ & $0.94(0.44$ to 2.01$)$ & $1.22(0.55$ to 2.70$)$ \\
\hline Girls & $\begin{array}{c}1.6(6 / 137245) \\
(n=1045)\end{array}$ & $\begin{array}{c}3.5(27 / 279399) \\
(n=2137)\end{array}$ & $0.46(0.19$ to 1.11$)$ & $0.51(0.21$ to 1.25$)$ & $0.46(0.19$ to 1.11$)$ & $0.51(0.21$ to 1.25$)$ \\
\hline All & $\begin{array}{c}2.1(16 / 279586) \\
(n=2129)\end{array}$ & $\begin{array}{c}3.1(48 / 562331) \\
(n=4288)\end{array}$ & 0.67 (0.38 to 1.19$)$ & $0.80(0.45$ to 1.43$)$ & 0.67 (0.38 to 1.19$)$ & $0.80(0.45$ to 1.43$)$ \\
\hline \multicolumn{7}{|l|}{ 9-36 months: } \\
\hline Boys & $\begin{array}{c}1.2(26 / 762500) \\
(n=1047)\end{array}$ & $\begin{array}{c}1.3(54 / 1506371) \\
(n=2080)\end{array}$ & 0.95 (0.60 to 1.52$)$ & 0.99 (0.62 to 1.59$)$ & $0.78(0.47$ to 1.28$)$ & $0.79(0.48$ to 1.31$)$ \\
\hline Girls & $\begin{array}{c}1.1(23 / 744638) \\
(n=1012)\end{array}$ & $\begin{array}{c}1.6(63 / 1476049) \\
(n=2053)\end{array}$ & $0.72(0.45$ to 1.17$)$ & 0.73 (0.45 to 1.19$)$ & $0.64(0.39$ to 1.07$)$ & $0.65(0.39$ to 1.09$)$ \\
\hline All & $\begin{array}{c}1.2(49 / 1507138) \\
(n=2059)\end{array}$ & $\begin{array}{c}1.4(117 / 2982420) \\
(n=4133)\end{array}$ & $0.83(0.59$ to 1.16$)$ & 0.85 (0.61 to 1.19$)$ & $0.71(0.50$ to 1.01$)$ & $0.72(0.50$ to 1.03$)$ \\
\hline \multicolumn{7}{|c|}{ 4.5-36 months: } \\
\hline Boys & $\begin{array}{c}1.5(36 / 904841) \\
(n=1084)\end{array}$ & $\begin{array}{c}1.5(75 / 1789303) \\
(n=2151)\end{array}$ & 0.95 (0.64 to 1.42$)$ & 1.05 (0.70 to 1.57$)$ & $0.82(0.54$ to 1.25$)$ & $0.90(0.59$ to 1.37$)$ \\
\hline Girls & $\begin{array}{c}1.2(29 / 881883) \\
(n=1045)\end{array}$ & $\begin{array}{c}1.9(90 / 1755448) \\
(n=2137)\end{array}$ & $0.64(0.42$ to 0.98$)$ & 0.67 (0.44 to 1.02$)$ & $0.59(0.38$ to 0.91$)$ & 0.61 (0.39 to 0.95$)$ \\
\hline All & $\begin{array}{c}1.3(65 / 1786724) \\
(n=2129)\end{array}$ & $\begin{array}{c}1.7(165 / 3544751) \\
(n=4288)\end{array}$ & $0.78(0.59$ to 1.05$)$ & 0.84 (0.63 to 1.12$)$ & $0.70(0.52$ to 0.94$)$ & $0.74(0.55$ to 1.00$)$ \\
\hline
\end{tabular}

ITT=intention to treat analysis; PP=per-protocol analysis (between 4.5 and 9 months of age PP and ITT are the same analysis).

The study covered six contiguous districts in Bissau, and participants were enrolled by the same team at the three health centres covering these districts. The beneficial effect of early measles vaccination tended to be largest in Cuntum, which had not taken part in the first trial of vitamin A supplementation at birth (see below) (web table D). We adjusted the main results for district by stratification; web table $\mathrm{D}$ shows unadjusted estimates. Little difference existed between adjusted and unadjusted estimates.

Interactions with campaigns and other interventions

The cohort was exposed to a large number of campaigns (table 1). We found no sign of differential participation in these campaigns. We therefore did the analyses censoring for participation in the campaigns one by one to see whether this modified the effect of the intervention. In general, censoring had no effect on the estimated effect of early two dose measles vaccine from 4.5 to 36 months of age (web table A).

We also tested whether the effect of early measles vaccine was the same within the treatment arms of concurrent trials. That was not the case within the trials of vitamin A supplementation at birth. A total of 5142 study children had taken part in these trials. The mortality rate ratio for early measles vaccine versus measles vaccine at 9 months of age differed significantly according to status in the trials of vitamin A supplementation at birth (web tables $\mathrm{E}$ and $\mathrm{F})(\mathrm{P}=0.011$, test for interaction of vitamin A supplementation at birth and early measles vaccine). In the intention to treat analysis, the mortality rate ratio for early measles vaccination was 1.07 (0.71 to 1.61) for the 3015 children who had received vitamin A supplementation at birth: 0.82 (0.44 to 1.53) for girls and 1.34 (0.78 to 2.29) for boys. Among the 2127 children who had received placebo at birth, the mortality rate ratio was 0.45 ( 0.24 to 0.84$): 0.40(0.17$ to 0.96$)$ for girls and 0.52 (0.21 to 1.29 ) for boys. Compared with children who received placebo at birth, neonatal vitamin A was associated with 2.5-fold higher mortality for children who received measles vaccine at 4.5 and 9 months of age (mortality rate ratio 2.59 (1.24 to 5.42$)$ ). The 1275 children not enrolled in the trials of vitamin A supplementation at birth had lower weight at enrolment and higher mortality during follow-up; this group included children who had travelled and received the first BCG

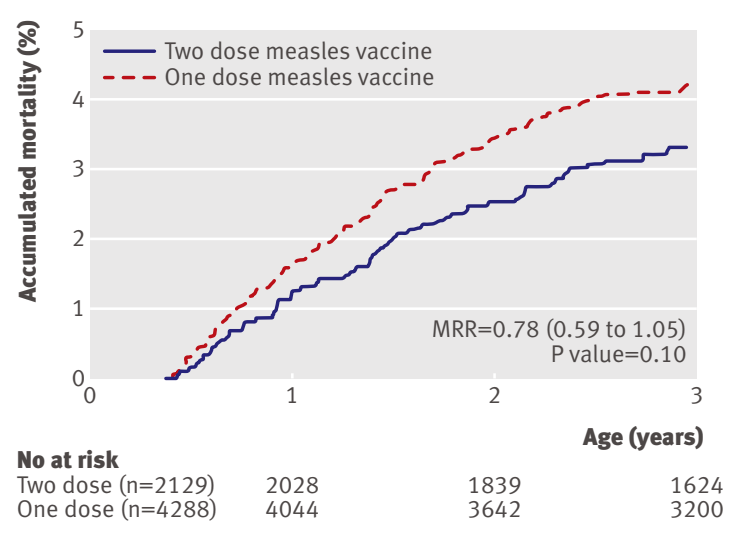

\begin{tabular}{l|l}
\hline Fig 2 & Kaplan-Meier accumulated mortality curves according
\end{tabular} to vaccination group in intention to treat analysis. MRR=mortality rate ratio 
vaccination outside the study area, and more twins and low birthweight children were included in this group as the vitamin A supplementation trials initially included only normal birthweight children. The mortality rate ratio of early measles vaccine versus measles vaccine at 9 months of age in this group of children was $0.74(0.42$ to 1.30$)$; the ratio was 0.71 (0.33 to 1.51 ) for girls and 0.79 ( 0.35 to 1.80 ) for boys (web table G). Vitamin A supplementation at birth is not official WHO policy, so we did the survival analysis of measles vaccine separately for the 3402 children who had not received vitamin A supplementation at birth - that is, the children who had received placebo in the vitamin A supplementation trials and the children who had not participated.

Main results for children who did not receive vitamin $A$ at birth-Between 4.5 and 36 months of age, the early two dose measles vaccine group (A) compared with Edmonston-Zagreb measles vaccine (group B) had mortality rate ratios of 0.59 ( 0.37 to 0.93$)$ in the intention to treat analysis and $0.49(0.30$ to 0.80$)$ in the per protocol analysis (web table $\mathrm{H}$ ). Compared with Schwarz measles vaccine (group $\mathrm{C}$ ), the mortality rate ratios were 0.59 $(0.37$ to 0.94$)$ in the intention to treat analysis and 0.51 (0.32 to 0.84 ) in the per protocol analyses (web table I). Combining the two standard measles vaccine groups, the mortality rate ratios were $0.59(0.39$ to 0.89$)$ in the intention to treat analysis and $0.50(0.32$ to 0.78$)$ in the per protocol analysis. Control for background factors (table 1) had no effect on the mortality estimate for early measles vaccine in the subgroup analysis of children who had not received vitamin A at birth.

Secondary results for children who did not receive vitamin $A$ at birth - In the intention to treat and per protocol analyses, the mortality rate ratio was 0.33 (0.13 to 0.86 ) between 4.5 and 9 months and 0.68 (0.43 to 1.08) and 0.56 ( 0.34 to 0.93 ) between 9 and 36 months of age (table 3). In the intention to treat analysis, the effect between 4.5 and 36 months of age was statistically significant for girls (mortality rate ratio 0.54 (0.31 to 0.95$)$ ) but not for boys (mortality rate ratio 0.65 (0.36 to 1.20$)$ ) (female:male estimate ratio 0.83 (0.36 to 1.90$) ; \mathrm{P}=0.65$ ). Similarly, in the per protocol analysis, the effect was significant for girls (mortality rate ratio 0.47 (0.26 to 0.86$)$ ) but not for boys (mortality rate ratio 0.55 (0.29 to 1.06$)$ ) (female:male estimate ratio 0.84 ( 0.35 to 2.04$) ; \mathrm{P}=0.71$ ) (table 3). The effect did not differ by season. After censoring for measles infection, the mortality rate ratios between 4.5 and 36 months of age became 0.65 ( 0.43 to 0.99$)$ in the intention to treat analysis and $0.55(0.35$ to 0.86 ) in the per protocol analysis. As subclinical measles infection at the start of the trial could have played a role, ${ }^{27}$ we did an analysis that included only the 2037 children born after the end of the measles epidemic (May 2004). The mortality rate ratios for early two dose measles vaccine versus measles vaccine at 9 months of age were 0.56 ( 0.30 to 1.04$)$ in the intention to treat analysis and 0.52 ( 0.28 to 0.99 ) in the per protocol analysis between 4.5 and 36 months of age in this group. Figure 3 shows that, although the mortality level was much lower for children born after the epidemic, the differential pattern in mortality for children receiving early two dose measles vaccine and standard measles vaccine at 9 months of age was the same in both cohorts.

\section{DISCUSSION}

This is one of the first trials of childhood vaccination with individual randomisation to measure the effect of a vaccine on all cause mortality. We had hypothesised a $25 \%$ reduction in mortality between 4.5 and 36 months of age but observed only a $22 \%$ (95\% confidence interval $-5 \%$ to $41 \%$ ) reduction, which was not statistically significant in the intention to treat analysis. The per protocol analysis suggested a $30 \%$ (6\% to $48 \%$ ) reduction in mortality. Among the secondary outcomes, the reduction in mortality between 4.5 and 36 months of age was statistically significant for girls in both the intention to treat and per protocol analyses. We did not find this effect for boys, but the difference in effect between girls and boys was not statistically significant. The study was not powered to test significant differential effects between subgroups in the secondary analyses. The analysis of possible interactions with other trials and campaigns suggested that vitamin A supplementation at birth may have a negative influence on the effect of early measles vaccination.

\section{Strengths and weaknesses}

This is the first trial designed to test a non-specific beneficial effect of measles vaccine that may provide some support for such effects, at least for girls. We have done one previous trial of two dose measles vaccine with the same objective, but it did not give similar results; this lack of effect was probably because we had not controlled for the effect of subsequent DTP vaccinations. ${ }^{25}$ To prevent this problem in this trial, we stipulated that the children should have received the third DTP vaccine before being enrolled. ${ }^{26} \mathrm{We}$ also considered in our analyses the children's participation in the major campaigns in the study area during the trial period.

A measles epidemic occurred at the beginning of the trial, and this could be considered both a strength and a weakness. It permitted us to document that early measles vaccination at 4.5 months of age was highly effective and would serve the targeted purpose of preventing measles infection. ${ }^{26}$ On the other hand, one could speculate that the non-specific beneficial effect of early measles vaccination was due to prevention of (undetected) cases of subclinical measles infection. However, subclinical measles infection may not be associated with increased mortality, ${ }^{33}$ and in this study we were able to show that the effect of early measles vaccination was the same in the cohort born after the measles epidemic (fig 3). Hence, consistent with many previous studies, ${ }^{57112434}$ the trial could be seen as supporting the hypothesis that measles vaccine has a beneficial effect on non-measles related childhood mortality.

From the perspective of a randomised trial, this study had several limitations, the most important of which was the lack of blinding of the vaccination status. Mothers bring children's vaccination cards to 


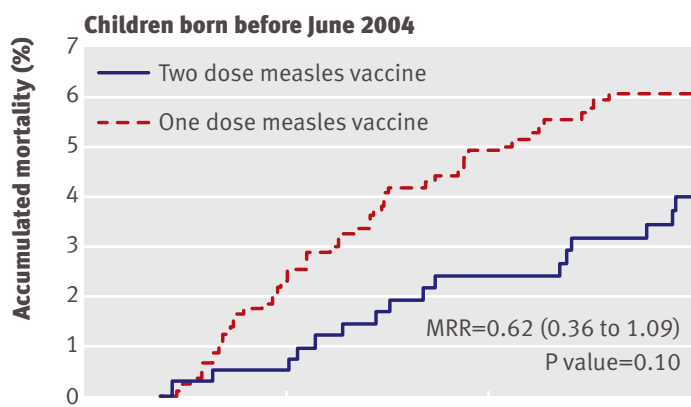

\section{No at risk}

Two dose

One dose

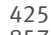

425
857

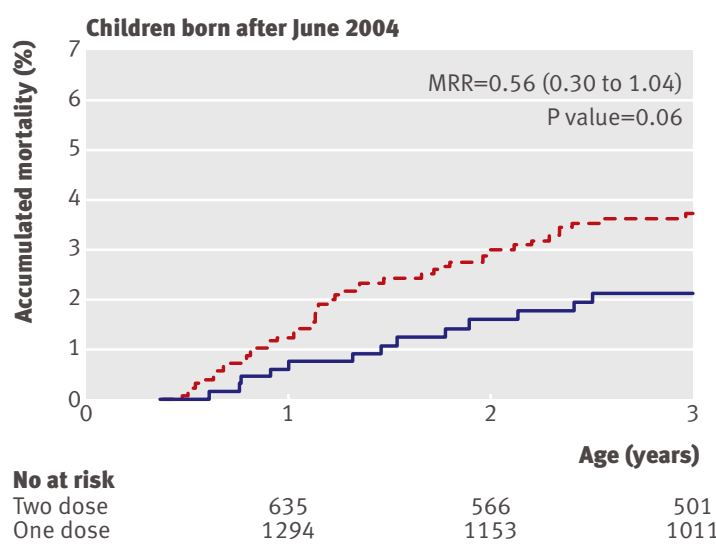

Fig 3 | Kaplan-Meier accumulated mortality curves according to vaccination group and birth cohort in intention to treat analysis in children without vitamin A supplementation at birth. MRR=mortality rate ratio. 1365 children in cohort born before June 2004: 444 randomised to two dose group and 921 to one dose group. 2037 children in cohort born after June 2004: 663 randomised to two dose group and 1374 to one dose group

consultations within the study area to facilitate identification and because it entitles their children to free consultations and essential drugs. However, the health workers, doctors, and nurses in the paediatric ward and at the health centres do not inspect the vaccination cards of sick children to guide the treatment, and they were not aware of the purpose of the study. Their clinical decisions are thus unlikely to have affected survival in a systematic way to the benefit of children who had received measles vaccine early. If vaccination status had affected treatment practice, it should also have affected the survival of children who had received measles vaccine at 4.5 months of age and vitamin $\mathrm{A}$ supplementation at birth, as the randomisation to vitamin A supplementation or placebo was blind. As this was not the case, we have no reason to think that biased clinical decisions affected the outcome of the trial.

Secondly, the study was not perfectly balanced with respect to drop-out. As we tested specific strains of measles vaccine, children who had received measles vaccine elsewhere before they attended the 9 month vaccination session were censored in the study because the strain and quality of their measles vaccine would not be known. We excluded more children at the
9 month vaccination session in groups $\mathrm{B}$ and $\mathrm{C}$ than in group A (fig 1). Assuming that these early measles vaccinations in groups $\mathrm{B}$ and $\mathrm{C}$ also had a beneficial effect, they would have lowered the mortality rate in group $\mathrm{B}$ and $\mathrm{C}$ and reduced the estimated beneficial effect of early measles vaccine (group A) between 4.5 and 9 months of age. On the other hand, censoring these children in the per protocol survival analysis may have removed a group with low mortality from groups $\mathrm{B}$ and $\mathrm{C}$ and therefore enhanced the estimated beneficial effect of group A between 9 and 36 months of age. This is part of the reason for differences between the per protocol and intention to treat analyses. Testing modifications in vaccination strategy for vaccines that are already available in routine use is difficult because the trial itself may have an effect on how the current routine vaccination is implemented. In this trial, the fact that we focused on measles vaccination and early measles vaccination may have meant that more children in the control group received early measles vaccination. The "true" estimate of the effect of early measles vaccination as a public health intervention may therefore be difficult to assess but is presumably somewhere between the intention to treat and per protocol estimates. For girls, for whom we hypothesised a strong beneficial effect, both intention to treat and per protocol estimates were statistically significant.

Thirdly, the cohort was affected by many other interventions, as is common in low income countries. From a public health perspective, examining the potential effect of these other interventions on the trial intervention would seem to be important.

\section{Critical features of intervention}

As regards the intervention, firstly, as pointed out above, the results depend on the fact that all children received the third DTP vaccine before entering the trial. ${ }^{26}$ Secondly, we also ensured that a gap of four weeks or more existed between the third administration of DTP vaccine and enrolment in the trial. Previous studies have suggested that a short interval between these vaccines is associated with increased mortality, ${ }^{35}$ and administration of measles vaccine and DTP vaccine at the same time has been linked to negative health outcomes. $^{36-38}$ Thirdly, these results apply only to Edmonston-Zagreb measles vaccine, ${ }^{39}$ which may be more effective in the presence of maternal antibodies than is the Schwarz stain of measles vaccine. ${ }^{13}$

We must emphasise that the beneficial effect for girls is related to these conditions. Measles vaccine will not necessarily always have a beneficial effect. ${ }^{36-38}$ Fortunately, this trial suggests that the beneficial effect of early two dose measles vaccine for girls is affected only to a limited extent by season and by other vaccination and vitamin A supplementation campaigns. However, vitamin A supplementation at birth may negate the beneficial effect of early measles vaccine.

\section{Consistency with previous studies}

For children who had not received vitamin A at birth, this trial is consistent with studies done in the 1970s and 
Table $3 \mid$ Mortality rates and mortality rate ratios of recipients of early two dose measles vaccine (group A) compared with measles vaccine at 9 months (groups $B+C$ ) among children who had not received vitamin A supplementation at birth $(n=3402)$

\begin{tabular}{|c|c|c|c|c|c|c|}
\hline \multirow[b]{2}{*}{ Age interval } & \multicolumn{2}{|c|}{$\begin{array}{l}\text { ITT mortality rate per } 100 \text { person years (deaths/ } \\
\text { person days) }\end{array}$} & \multirow[b]{2}{*}{$\begin{array}{l}\text { ITT mortality rate } \\
\text { ratio }(A /(B+C))\end{array}$} & \multirow[b]{2}{*}{$\begin{array}{l}\text { ITT mortality rate ratio } \\
(A /(B+C)) \text { (with censoring } \\
\text { for measles infection) }\end{array}$} & \multirow[b]{2}{*}{$\begin{array}{l}\text { PP mortality rate ratio } \\
(A /(B+C))\end{array}$} & \multirow[b]{2}{*}{$\begin{array}{l}\text { PP mortality rate ratio } \\
(A /(B+C)) \text { (with censoring } \\
\text { for measles infection) }\end{array}$} \\
\hline & $\begin{array}{l}\text { Early two dose measles } \\
\text { vaccine (group A) }\end{array}$ & $\begin{array}{l}\text { Measles vaccine } \\
\text { at } 9 \text { months } \\
\text { (groups B and C) }\end{array}$ & & & & \\
\hline \multicolumn{7}{|l|}{ 4.5-9 months: } \\
\hline Boys & $\begin{array}{c}1.5(3 / 73603) \\
(n=558)\end{array}$ & $\begin{array}{c}3.7(15 / 147459) \\
(n=1130)\end{array}$ & $0.36(0.10$ to 1.26$)$ & 0.47 (0.13 to 1.72$)$ & $0.36(0.10$ to 1.26$)$ & 0.47 (0.13 to 1.72$)$ \\
\hline Girls & $\begin{array}{c}1.0(2 / 71959) \\
(n=549)\end{array}$ & $\begin{array}{c}3.4(14 / 152480) \\
(n=1165)\end{array}$ & 0.30 (0.07 to 1.31$)$ & 0.37 (0.08 to 1.68$)$ & $0.30(0.07$ to 1.31$)$ & 0.37 (0.08 to 1.68$)$ \\
\hline All & $\begin{array}{c}1.3(5 / 145562) \\
(n=1107)\end{array}$ & $\begin{array}{c}3.5(29 / 299939) \\
(n=2295)\end{array}$ & 0.33 (0.13 to 0.86$)$ & 0.42 (0.16 to 1.12 ) & 0.33 (0.13 to 0.86$)$ & 0.42 (0.16 to 1.12$)$ \\
\hline \multicolumn{7}{|l|}{ 9-36 months: } \\
\hline Boys & $\begin{array}{c}1.0(11 / 401246) \\
(n=540)\end{array}$ & $\begin{array}{c}1.3(27 / 783132) \\
(n=1086)\end{array}$ & 0.79 (0.39 to 1.59$)$ & $0.86(0.42$ to 1.75$)$ & 0.64 (0.30 to 1.38$)$ & $0.68(0.31$ to 1.46$)$ \\
\hline Girls & $\begin{array}{c}1.2(13 / 386943) \\
(n=531)\end{array}$ & $\begin{array}{c}2.0(43 / 794503) \\
(n=1117)\end{array}$ & 0.62 (0.33 to 1.15$)$ & 0.63 (0.34 to 1.18$)$ & 0.52 (0.27 to 1.02$)$ & 0.53 (0.27 to 1.04$)$ \\
\hline All & $\begin{array}{c}1.1(24 / 788189) \\
(n=1071)\end{array}$ & $\begin{array}{c}1.6(70 / 1577635) \\
(n=2203)\end{array}$ & 0.68 (0.43 to 1.08 ) & 0.71 (0.45 to 1.14$)$ & 0.56 (0.34 to 0.93$)$ & $0.58(0.35$ to 0.96$)$ \\
\hline \multicolumn{7}{|c|}{ 4.5-36 months: } \\
\hline Boys & $\begin{array}{c}1.1(14 / 474849) \\
(n=558)\end{array}$ & $\begin{array}{c}1.6(42 / 930591) \\
(n=1130)\end{array}$ & 0.65 (0.36 to 1.20$)$ & 0.76 (0.41 to 1.41$)$ & 0.55 (0.29 to 1.06$)$ & 0.63 (0.33 to 1.22 ) \\
\hline Girls & $\begin{array}{c}1.2(15 / 458902) \\
(n=549)\end{array}$ & $\begin{array}{c}2.2(57 / 946983) \\
(n=1165)\end{array}$ & 0.54 (0.31 to 0.95$)$ & 0.58 (0.33 to 1.03$)$ & $0.47(0.26$ to 0.86$)$ & $0.50(0.27$ to 0.92$)$ \\
\hline All & $\begin{array}{c}1.1(29 / 933751) \\
(\mathrm{n}=1107)\end{array}$ & $\begin{array}{c}1.9(99 / 1877574) \\
(n=2295)\end{array}$ & 0.59 (0.39 to 0.89$)$ & 0.65 (0.43 to 0.99$)$ & $0.50(0.32$ to 0.78$)$ & $0.55(0.35$ to 0.86$)$ \\
\hline
\end{tabular}

ITT=intention to treat analysis; PP=per protocol analysis (between 4.5 and 9 months of age PP and ITT are the same analysis).

1980s, which suggested that measles vaccine reduced mortality by $40 \%$ or more. ${ }^{1-7}$ These studies were done before DTP vaccination was introduced and before vitamin A supplementation was used. Our trial suggests at least two different pathways by which early two dose measles vaccine may reduce mortality.

Firstly, in the age group from 4.5 to 9 months of age, the children randomised to measles vaccine tended to have lower mortality than the children who had the third DTP vaccine as their most recent vaccine. A few previous randomised trials have supported the hypothesis that early measles vaccine was associated with lower mortality before the age of standard measles vaccination. In a small randomised trial in Sudan, in which children were given measles vaccine at 5 months of age and controls received meningococcal vaccines, the children vaccinated for measles had a $91 \%$ lower mortality until the control children received measles vaccine at 9 months of age (mortality rate ratio 0.09 (0.01 to 0.71$)) .{ }^{40}$ In Bissau, we did an unplanned randomised trial owing to a war that stopped all vaccinations: the children who had been randomised to measles vaccine at 6 months just before the war had $70 \%$ lower mortality during the war than did the children who had been randomised to inactivated polio vaccine at 6 months of age (mortality rate ratio $0.30(0.08$ to 0.87$)) .{ }^{24}$ In these studies, DTP vaccine was not administered at the same time as measles vaccine, ${ }^{12240}$ and the reduction in mortality was not related to prevention of measles infection. ${ }^{2440}$

Secondly, although the results did not reach statistical significance, having received two doses by 9 months of age may also have been associated with better survival between 9 months and 36 months of age than having the first measles vaccine at 9 months of age as currently recommended. Previous studies have also indicated that two doses of measles vaccine by 9 months may be better than just one dose at 9 months. We used measles vaccine at 6 to 8 months of age in campaigns in Bissau before 9 months was defined as the official WHO policy. Campaigns were annual or biannual, so whether a child was vaccinated at 6-8 or 9-11 months of age depended on the child's birthday in relation to when the campaign was arranged and not on maternal selection bias. From 9 months to 5 years of age, the children vaccinated at 6-8 months had significantly lower mortality compared with children who were vaccinated at 9-11 months of age. This beneficial effect was particularly strong among children who received measles vaccine twice; the mortality rate ratio was 0.41 ( 0.19 to $0.85) .{ }^{41}$ More research is clearly needed into the possible mechanisms that could explain this observation.

Although vitamin A supplementation is administered to reduce vitamin A deficiency, we have shown in several observational and randomised studies in Guinea-Bissau and Ghana that vitamin A may interact with vaccinations and enhance the non-specific effects of vaccines. ${ }^{30-3242-44}$ Vitamin A may interact beneficially with measles vaccine administered alone and negatively with DTP vaccine. ${ }^{303242-44}$ Unexpectedly, in this trial neonatal vitamin A compared with placebo at birth was associated with twofold higher mortality in children who received measles vaccine at 4.5 months of age. On the basis of previous studies that showed a 


\section{WHAT IS ALREADY KNOWN ON THIS TOPIC}

Vaccines may have non-specific and sex differential effects on all cause mortality

Many observational studies have suggested that standard titre measles vaccine has beneficial non-specific effects on mortality, particularly for girls

Re-analyses of measles vaccination trials have suggested that diphtheria-tetanus-pertussis (DTP) vaccination has negative effects when administered after measles vaccination

\section{WHAT THIS STUDY ADDS}

An early two dose measles vaccination strategy was associated with a non-significant $22 \%$ reduction in all cause mortality between 4.5 and 36 months of age in children who had received all doses of DTP vaccine before enrolment

The effect was statistically significant for girls, but the difference in the effect of the intervention between girls and boys was not statistically significant

Prevention of measles infection explained only a small part of the effect on overall mortality

beneficial effect of vitamin A given with measles vaccine,${ }^{42}$ we would have expected a beneficial effect. However, we have previously found that vitamin A administered simultaneously with measles vaccine and DTP vaccine produced a negative effect. ${ }^{42}$ The response may have been changed in this trial because only four weeks passed between the third DTP vaccine and measles vaccination, whereas the gap between these vaccines would normally be $4-5$ months. In any event, this trial and several of our previous studies indicate that immunomodulating interventions may interact and have major unexpected effects on childhood survival in low income countries. ${ }^{1930-3242-44}$

\section{Interpretation}

Although it showed no overall effect in the main analysis, this trial suggested a non-specific beneficial effect of measles vaccine among girls who have not received neonatal vitamin $\mathrm{A}$. When the overall effect of measles vaccine is compared with the effect after censoring for measles infection, only around $10-20 \%$ of the reduction in mortality seems to be due to the prevention of infection with measles and deaths from measles. This is consistent with the many previous observational studies that have shown that censoring for measles infection had little influence on the estimate of the effect of measles vaccine on total mortality. ${ }^{11}$ The observed beneficial effect of early measles vaccine in girls thus cannot be explained by the prevention of cases of measles and deaths from measles.

When a strong effect of measles vaccine on childhood survival was reported in observational studies and community intervention trials in the 1980s and 1990s, this was assumed to be due to selection bias or the fact that measles vaccine prevented not only mortality from acute measles but also the long term negative consequences of measles infection. ${ }^{945}$ We subsequently found that measles had no long term negative consequences in Guinea-Bissau, Senegal, and Bangladesh. ${ }^{33344647}$ Many factors suggest that selection bias is an unlikely explanation for the remarkable effect of measles vaccine on mortality in observational studies. ${ }^{71124}$
The way to explain the beneficial non-specific effects of measles vaccine for girls is that early measles vaccine reduces susceptibility to severe infections with pathogens other than measles virus. In this trial, children vaccinated against measles had a significant reduction in admission to hospital, particularly admissions due to pneumonia, and this effect was strongest for girls (unpublished data). We have previously observed that the non-specific effects of vaccines seem to be strongest for pneumonia. ${ }^{48}$

Very few studies have examined the immunological mechanisms that might explain the non-specific effects of vaccines, although studies of heterologous immunity in animals have shown that priming with one antigen may either enhance or reduce susceptibility to subsequent infection with unrelated antigens. ${ }^{490}$ The observation that girls who received measles vaccine at 4 . 5 months of age had a reduction in mortality compared with girls who had the third DTP vaccine as their most recent vaccine until 9 months of age could be related to differences in immune priming of live and inactivated vaccines. In animal studies of live and inactivated vaccines of the same antigen, the live vaccine has generally been associated with a better capacity to handle a subsequent challenge. ${ }^{51-55}$ Little is known about how these effects influence the reaction to unrelated antigens.

\section{Implications}

WHO's Global Advisory Committee on Vaccine Safety has declared that it will keep a watch on the non-specific effects of vaccines and has supported the proposition that only randomised studies will provide convincing evidence for or against non-specific effects of vaccines on mortality. ${ }^{56}$ This posed a dilemma, as randomising children to vaccines that are already in the routine schedule is not usually considered acceptable. However, by studying the early administration of measles vaccine, we have been able to test the possible non-specific effects of measles vaccine in a randomised trial. Measles vaccine tended to be associated with a beneficial non-specific effect, as we had hypothesised, but the overall result did not reach statistical significance and should be interpreted with caution. However, we had also hypothesised that the effects would be strongest for girls, and the trial suggests that beneficial non-specific effects of measles vaccine are likely for girls.

Surprisingly, we found an interaction between vitamin A supplementation at birth and the effect of early measles vaccination on mortality between 4.5 and 36 months of age; the beneficial effect was much stronger among children who had not received vitamin $\mathrm{A}$ at birth. As vitamin A at birth is not WHO policy, further studies are needed to examine the possibility that we may achieve substantial reductions in childhood mortality in low income countries by giving an additional dose of measles vaccine before 9 months of age to children who have not received vitamin A at birth.

Contributors: MLG, CLM, HCW, IML, and PA designed the study. CLM, M$L G$ and PA initiated the study routines. CLM and CB treated the measles patients during the outbreak. AR supervised the routine registration system. CSB was principle investigator of the vitamin A trials and 
provided the data on these children. Data were analysed by PA, AA, and $H R$. AA and HR were responsible for the statistical analyses. PA wrote the first draft of the paper, and all authors contributed to the final version. PA is the guarantor.

Funding: The research on the decline in maternal antibodies that inspired this trial was funded by the Thrasher Foundation. This study was mostly funded by DANIDA and the Danish National Research Foundation. The project also received support from Fonden til Lægevidenskabens Fremme and Novo Nordisk Foundation. The Bandim Health Project received support from DANIDA. PA holds a research professorship grant from the Novo Nordisk Foundation. The funding agencies had no role in the study design, data collection, data analysis, data interpretation, or the writing of the report.

Competing interests: All authors have completed the Unified Competing Interest form at www.icmje.org/coi_disclosure.pdf (available on request from the corresponding author) and declare: no support from any organisation for the submitted work; no financial relationships with any organisations that might have an interest in the submitted work in the previous 3 years; no other relationships or activities that could appear to have influenced the submitted work.

Ethical approval: The protocol was approved by the Danish Central Ethical Committee, the Gambia/MRC scientific and ethics committees, and the Guinean Ministry of Health's Research Coordination Committee. Data sharing: No additional data available.

1 Kasongo Project Team. Influence of measles vaccination on survival pattern of 7-35-month-old children in Kasongo, Zaire. Lancet 1981;1:764-7.

2 Aaby P, Bukh J, Lisse IM, Smits AJ. Measles vaccination and child mortality. Lancet 1981;ii:93.

3 Aaby P, Bukh J, Lisse IM, Smits AJ. Measles vaccination and reduction in child mortality: a community study from Guinea-Bissau. J Infect 1984;8:13-21.

4 Desgrées du Loû A, Pison G, Aaby P. The role of immunizations in the recent decline in childhood mortality and the changes in the female/ male mortality ratio in rural Senegal. Am J Epidemio 1995;142:643-52.

5 Aaby P, Samb B, Simondon F, Knudsen K, Coll Seck AM, Bennett J, et al. Divergent mortality for male and female recipients of low-titre and high-titre measles vaccines in rural Senegal. Am J Epidemiol 1993;138:746-55.

6 Aaby P, Bukh J, Lisse IM, Smits AJ, Gomes J, Fernandes MA, et al. Determinants of measles mortality in a rural area of Guinea-Bissau: crowding, age, and malnutrition. J Trop Pediatr 1984;30:164-9.

7 Aaby P, Pedersen IR, Knudsen K, da Silva MC, Mordhorst CH, Helm-Petersen NC, et al. Child mortality related to seroconversion or lack of seroconversion after measles vaccination. Pediatr Infect Dis J 1989;8:197-200

8 Holt EA, Boulos R, Halsey NA, Boulos IM, Boulos C. Childhood survival in Haiti: protective effect of measles vaccination. Pediatrics 1990;85:188-94

9 Clemens ID, Stanton BF, Chakraborty J, Chowdhury S, Rao M, Ali M. Measles vaccination and childhood mortality in rural Bangladesh. Am J Epidemiol 1988;128;1330-9.

10 Koenig MA, Khan MA, Wojtyniak B, Clemens JD, Chakraborty Fauveau $V$, et al. The impact of measles vaccination upon childhood mortality in Matlab, Bangladesh. Bull World Health Organ 1990;68:441-7.

11 Aaby P, Samb B, Simondon F, Coll Seck AM, Knudsen K, Whittle H. Non-specific beneficial effect of measles immunisation: analysis of mortality studies from developing countries. BMJ 1995;311:481-5.

12 Expanded Programme on Immunisation. The optimal age for measles immunization. Wkly Epidemiol Rec 1982;57:89-91.

13 Whittle H, Hanlon P, O’Neill K, Hanlon L, Marsh V, Jupp E, et al. Trial of high-dose Edmonston-Zagreb measles vaccine in the Gambia: antibody response and side-effects. Lancet 1988;2:811-4.

14 Whittle H, Campbell H, Rahman S, Armstrong JRM. Antibody persistence in Gambian children after high-dose Edmonston-Zagreb measles vaccine. Lancet 1990;336:1048-51.

15 Aaby P, Jensen TG, Hansen HL, Kristiansen H, Thårup J, Poulsen A, et al. Trial of high-dose Edmonston-Zagreb measles vaccine in Guinea-Bissau: protective efficacy. Lancet 1988;2:809-11.

16 Aaby $\mathrm{P}$, Knudsen $\mathrm{K}$, Whittle $\mathrm{H}$, Lisse IM, Thårup J, Poulsen $\mathrm{A}$, et al. Long-term survival after Edmonston-Zagreb measles vaccination in Guinea-Bissau: increased female mortality rate. J Pediatr 1993;122:904-8.

17 Aaby P, Samb B, Simondon F, Knudsen K, Coll Seck AM, Bennett J, et al. Sex-specific differences in mortality after high titre measles immunization in rural Senegal. Bull World Health Organ 1994;72:761-70.
18 Knudsen KM, Aaby P, Whittle H, Rowe M, Samb B, Simondon F, et al Child mortality following standard, medium or high titre measles mmunization in West Africa. Int J Epidemiol 1996;25:665-73.

19 Aaby P, Jensen H, Samb B, Cisse B, Sodeman M, Jakobsen M, et al. Differences in female-male mortality after high-titre measles vaccine and association with subsequent vaccination with diphtheriatetanus-pertussis and inactivated poliovirus: reanalysis of West African studies. Lancet 2003;361:2183-8.

20 Holt EA, Moulton LH, Siberry GK, Halsey NA. Differential mortality measles vaccine titer and sex. J Infect Dis 1993;168:1987-96.

21 Expanded Programme on Immunization. Safety of high titre measles vaccines. Wkly Epidemiol Rec 1992;67:357-61.

22 Garly ML, Martins CL, Balé C, da Costa F, Dias F, Whittle H, et al. Early two-dose measles vaccination schedule in Guinea-Bissau: good protection and coverage in infancy. Int J Epidemiol 1999;28:347-52.

23 Garly ML, Balé C, Martins C, Monteiro M, George E, Kidd M, et al. Measles antibody responses after early two dose trials in GuineaBissau with Edmonston-Zagreb and Schwarz standard-titre measles vaccine: better antibody increase from booster dose of the Edmonston-Zagreb vaccine. Vaccine 2001;19:1951-9.

24 Aaby P, Garly ML, Balé C, Martins C, Jensen H, Lisse IM, et al. Survival of previously measles-vaccinated and measles-unvaccinated children in an emergency situation: an unplanned study. Pediatr Infect Dis / 2003;22:798-805.

25 Aaby P, Garly ML, Jensen H, Martins C, Balé C, Benn CS, et al. Inactivated polio and diphtheria-tetanus-pertussis vaccines associated with increased female mortality prior to measles vaccination: observations from vaccination trials in Guinea-Bissau. Pediatr Infect Dis J 2007;26:247-52.

26 Martins CL, Garly ML, Balé C, Rodrigues A, Ravn H, Whittle HC, et al. Protective efficacy of standard Edmonston-Zagreb measles vaccination in infants aged 4.5 months: interim analysis of a randomised clinical trial. BMJ 2008;337:a661.

27 Martins C, Bale C, Garly ML, Rodrigues A, Lisse IM, Andersen A, et al. Girls may have lower levels of maternal measles antibodies and higher risk of subclinical measles infection before the age of measles vaccination. Vaccine 2009;27:5220-5.

28 Aaby P. Bandim: an unplanned longitudinal study. In: Das Gupta M, Aaby P, Pison G, Garenne M, eds. Prospective community studies in developing countries. Oxford University Press, 1997:276-96.

29 Aaby P, Martins C, Bale C, Garly ML, Rodrigues A, Biai S, et al. Sex differences in the effect of vaccines on the risk of hospitalisation due to measles in Guinea-Bissau. Pediatr Infect Dis J 2010;29:324-8.

30 Benn CS, Fisker A, Roth A, Diness BR, Lausch KR, Ravn H, et al. Randomised trial of vitamin A supplementation and $B C G$ vaccination at birth to low-birth-weight children. BMJ 2010;340:c1101.

31 Benn CS, Diness BR, Roth A, Nante E, Fisker AB, Lisse IM, et al. Effect of 50,000 IU vitamin $A$ given with $B C G$ vaccine on mortality in infants in Guinea-Bissau: randomised placebo controlled trial. BMJ 2008;336:1416-20.

32 Benn CS, Rodrigues A, Yazdanbakhsh M, Fisker AB, Ravn H, Whittle $\mathrm{H}$, et al. The effect of high-dose vitamin A supplementation administered with BCG vaccine at birth may be modified by subsequent DTP vaccination. Vaccine 2009;27:2891-8.

33 Aaby P, Samb B, Simondon F, Cisse B, Jensen H, Lisse IM, et al. Low mortality after mild measles infection compared to uninfected children in rural West Africa. Vaccine 2002;21:120-6.

34 Aaby P, Bhuyia A, Nahar L, Knudsen K, Francisco A, Strong M. The survival benefit of measles immunisation may not be explained entirely by the prevention of measles disease. Int J Epidemiol 2003;32:106-15.

35 Garly ML, Trautner SL, Marx C, Danebod K, Nielsen J, Ravn H, et al. Thymus size at 6 months of age and subsequent child mortality. Pediatr 2008;153:683-8.

36 Aaby P, Jensen H, Walraven G. Age-specific changes in female-male mortality ratio related to the pattern of vaccinations: an observational study from rural Gambia. Vaccine 2006;24:4701-8.

37 Aaby P, Vessari H, Nielsen J, Maleta K, Benn CS, Jensen H, et al. Nonspecific and sex-differential effects of routine immunizations in rural Malawi. Pediatr Infect Dis / 2006;25:721-7.

38 Aaby P, Benn CS. Assessment of childhood immunisation coverage? Lancet 2009;373:1428.

39 Bennett JV, Cutts FT, Katz SL. Edmonston-Zagreb measles vaccine: a good vaccine with an image problem. Pediatrics 1999;104:1123-4.

40 Aaby $\mathrm{P}$, Ibrahim S, Libman $\mathrm{M}$, Jensen $\mathrm{H}$. The sequence of vaccinations and increased female mortality after high-titre measles vaccine: trials from rural Sudan and Kinshasa. Vaccine 2006;24:2764-71.

41 Aaby P, Andersen M, Sodemann M, Jakobsen M, Gomes J, Fernandes M. Reduced childhood mortality after standard measles vaccination at 4-8 months compared with 9-11 months of age. BM 1993;307:1308-11.

42 Benn CB, Martins C, Rodrigues A, Fisker AB, Christoffersen D, Aaby P. The effect of vitamin A supplementation administered with missing 
vaccines during national immunisation days in Guinea-Bissau. Int J Epidemiol 2009;38:304-11.

43 Benn CS, Aaby P, Nielsen J, Binka FN, Ross DA. Does vitamin A supplementation interact with routine vaccinations? An analysis of the Ghana vitamin A supplementation trial. Am J Clin Nutr 2009;90:629-39.

44 Roth A, Benn CB, Ravn H, Rodrigues A, Lisse IM, Yazdanbakhsh M, et al. A randomised trial of the effect of revaccination with BCG in early childhood and mortality. BMJ 2010;340:C809.

45 Cooper WO, Boyce TG, Wright PF, Griffin MR. Do childhood vaccines have non-specific effects on mortality? Bull World Health Organ 2003;81:821-6.

46 Aaby P, Lisse I, Mølbak K, Knudsen K, Whittle H. No persistent T lymphocyte immunosuppression or increased mortality after measles infection: a community study from Guinea-Bissau. Pediatr Infect Dis / 1996;15:39-44.

47 Aaby P, Samb B, Andersen M, Simondon F. No long-term excess mortality after measles infection: a community study from Senegal. Am J Epidemiol 1996;143:1035-41.

48 Veirum JE, Sodemann M, Biai S, Jakobsen M, Hedegaard K, Jensen H, et al. Routine vaccinations associated with divergent effects on female and male mortality at the paediatric ward in Bissau, GuineaBissau. Vaccine 2005;23:1197-204.

49 Shann F. Heterologous immunity and the non-specific effects of vaccines: a major medical advance? Pediatr Infect Dis J 2004;23:555-8.
50 Welsh RM, Selin LH. No one is naïve: the significance of heterologus T-cell immunity. Nat Rev Immunol 2002;2:417-26.

51 Lindblad EB, Elhay MJ, Silva R, Appelberg R, Andersen P. Adjuvant modulation of immune responses to tuberculosis subunit vaccines. Infect Immun 1997;65:623-9.

52 Barnard A, Mahon BP, Watkins J, Redhead K, Mills KH. Th1/Th2 cell dichotomy in acquired immunity to Bordetella pertussis: variables in the in vivo priming and in vitro cytokine detection techniques affect the classification of T-cell subsets as Th1, Th2 or Th0. Immunology 1996;87:372-80.

53 Graham BS, Henderson GS, Tang YW, Lu X, Neuzil KM, Colley DG. Priming immunization determines Thelper cytokine mRNA expression patterns in lungs of mice challenged with respiratory syncytial virus. J Immunol 1993;151:2032-40.

54 Fischer JE, Johnson JE, Johnson TR, Graham BS. Pertussis toxin sensitization alters the pathogenesis of subsequent respiratory syncytial virus infection. J Infect Dis 2000;182:1029-38.

55 Moran TM, Park H, Fernandez-Sesma, Schulman JL. Th2 responses to inactivated Influenza virus can be converted to Th1 responses and facilitate recovery from heterosubtypic virus infection. J Infect Dis 1999;180:579-85.

56 Meeting of Global Advisory Committee on Vaccine Safety, 18-19 June 2008. Wkly Epidemiol Rec 2008;83:287-92.

Accepted: 20 September 2010 